\title{
Impact of vitamin D supplementation on cardio-metabolic status and androgen profile in women with polycystic ovary syndrome: placebo-controlled clinical trial
}

Nearmeen M. Rashad ${ }^{1 *}$ (D, Azza H. Abd El-Fatah ${ }^{1}$, Mohamed El-Bakry Lashin², Hala G. Abomandour ${ }^{3}$ and Reem M. Allam ${ }^{4}$

\begin{abstract}
Background: Polycystic ovary syndrome (PCOS) is a heterogeneous disorder of reproductive, endocrine, and metabolic functions. Vitamin D has an influence on metabolic and reproductive functions. This study was designed to explore the levels of free 25 hydroxycholecalciferol [25(OH)-D] in PCOS patients. We also aimed to clarify the impact of vitamin D supplementation on cardio-metabolic status, androgen profile, and clinical features of PCOS.

Results: Our results revealed significant lower levels of serum 25(OH)-D in PCOS women compared with healthy controls. Even more importantly, our results reported that 25(OH)-D levels were negatively correlated with cardiometabolic risk factors, androgenic profile, and clinical features of PCOS. Stepwise multiple linear regression analysis revealed that carotid intima-media thickness (CIMT), fasting serum insulin (FSI), and fasting plasma glucose (FPG) were the main predictors of $25(\mathrm{OH})$-D levels among other clinical and laboratory biomarkers. Considering the impact of VD supplementation in the PCOS group, there were significant improvements of cardio-metabolic risks, PCOS phenotype, and androgenic profile. Even more important, these results are associated with increasing $25(\mathrm{OH})$ -

D serum levels after VD supplementations. Logistic regression analysis observed that androstenedione, FSI, and hirsutism score were independent predictors of response to VD supplementation.

Conclusion: The supplementation of VD for 12 weeks improved the cardio-metabolic and androgenic profiles of PCOS. Furthermore, VD supplementation could be a promising treatment of PCOS and its associated morbidity in PCOS-deficient women.
\end{abstract}

Trial registration: NCT04117750

Keywords: Polycystic ovary syndrome, VD supplementation, Androgen profile, Cardio-metabolic

\section{Background}

Polycystic ovarian syndrome (PCOS) is a heterogeneous disorder affecting $5-10 \%$ of women of reproductive age [1]. It is a disorder that affects the reproductive, endocrine, and metabolic functions and is the leading cause of chronic anovulation leading to infertility [2].

PCOS is characterized by hyperandrogenism, chronic oligo or anovulation, and polycystic ovaries

\footnotetext{
* Correspondence: nrashad78@yahoo.com; n.rashad@zu.edu.eg ${ }^{1}$ Internal Medicine Department, Faculty of Medicine, Zagazig University, Zagazig, Egypt

Full list of author information is available at the end of the article
}

[3]. Hyperandrogenism, in particular, is a hallmark feature of PCOS because it is strongly implicated in the genesis of the disorder [4] and is also associated with metabolic derangements that contribute to the underlying pathophysiology [5]. Also, it is associated with cardiovascular risk factors including obesity, insulin resistance (IR), dyslipidemia, endothelial dysfunction, and metabolic syndrome $[2,5]$.

Vitamin D (VD) is a fat-soluble vitamin that is naturally present in very few foods and available as a dietary supplement. It is a steroid hormone with pleiotropic effects. In addition to the main effects of VD on the bone 
and calcium metabolism, it has other roles in the body, including modulation of cell growth, neuromuscular and immune function, and reduction of inflammation [6].

VD deficiency is now recognized as a pandemic disease. Its prevalence varies according to geographic location, season, ethnicity, and the standard laboratory value of what is considered normal, deficient, and insufficient. VD deficiency is a risk factor for hypertension, diabetes, and various cancers [7]. Accumulating evidences suggest that VD deficiency might be a causal factor in the pathogenesis of IR and the metabolic syndrome in PCOS [8]. Carotid intima-media thickness (CIMT) measured by ultrasound is a noninvasive, safe, low-cost, reproducible, and wellvalidated marker of preclinical atherosclerosis $[9,10]$.

PCOS is the most frequent endocrine disorder among women of a reproductive age and VD deficiency is a key problem in PCOS patients conversely; the basic mechanisms underlying the favorable effects of vitamin $\mathrm{D}$ in PCOS are still obscure. Resolving this mechanism may provide insight into the pathophysiology of this syndrome. It can also offer a new therapeutic option for PCOS women. Thus, the present study was designed to explore the levels of free 25 hydroxyvitamin D [25(OH)$\mathrm{D}]$ in PCOS patients. We also aimed to clarify the impact of vitamin D supplementation cardio-metabolic status, androgen profile, and clinical features of PCOS.

\section{Methods}

This placebo-controlled trial comprised 95 women with PCO recruited from Outpatient Clinics of the Endocrinology Unit of Internal Medicine and Obstetrics and Gynecology Departments, Faculty of Medicine, Zagazig University, Egypt, and 50 healthy women matched to PCOS women as regards age and ethnic origin.

The diagnosis of PCOS was based on the 2004 revised Rotterdam criteria [11]. All women underwent a menstrual history and thorough clinical examination. All patients were assessed at the study start on the third day of a spontaneous or progesterone-induced menstrual cycle. Anthropometric measures were estimated, including waist/hip ratio, height, and weight and then we calculated the body mass index (BMI). We estimated the hirsutism score according to Ferriman and Gallwey [12].

.Ovarian volume and antral follicular count (AFC) were evaluated by transvaginal ultrasound (TVS).

PCOS patients were randomized divided into an intervention group $(n=55)$ who received vitamin D supplements $(42,000 \mathrm{IU}$ oral vitamin D per week and $500 \mathrm{mg}$ calcium carbonate per day for 12 weeks) and nonintervention group $(n=40)$ who received $500 \mathrm{mg}$ calcium carbonate per day for 12 weeks.

The exclusion criteria for all women included a history of hyperandrogenic states (such as nonclassic congenital adrenal hyperplasia, androgen-secreting tumors, Cushing's syndrome, 21-hydroxylase deficiency, or hyperprolactinemia), DM, hypertension, liver, kidney, or thyroid diseases. In addition, subjects on non-steroidal anti-inflammatory drugs and multivitamins, as well as patients treated with hormone replacement therapy.

At the start of the study, the participants were asked to maintain their usual diet and level of physical activity throughout the study period as well as not to receive any lipid-lowering medications and medications that might affect their reproductive physiology during the 12-week intervention.

At baseline and at the endpoint of the 12 weeks of study, anthropometrical measurements were estimated and blood samples were collected for biochemical analyses. Written informed consent was taken from all of the participants after explaining details and benefits as well as risks to them. The ethical committee of the Faculty of Medicine, Zagazig University, approved our study protocol.

\section{Sampling of blood}

Blood samples were drawn from all subjects during the early follicular phase of the menstrual cycles. One milliliter was collected into tubes containing fluoride for fasting plasma glucose (FPG). A second remaining part underwent immediate serum separation and was stored at $-20^{\circ} \mathrm{C}$ until the serum analysis. Calcium, phosphate, and albumin were measured. Total cholesterol, highdensity lipoprotein (HDL) cholesterol, and triglyceride levels were determined using routine enzymatic methods (Spinreact, Girona, Spain). Low-density lipoprotein (LDL) cholesterol levels were calculated using the Friedewald formula [13].

\section{Immunochemical assays}

We measured prolactin, FSH, and LH levels via chemiluminescence immunoassays (CLIA) provided by (Immunospec Corporation, CA, USA). Serum high-sensitivity Creactive protein (hs-CRP) concentrations were measured using high sensitivity enzyme-linked immunosorbent assays (ELISA) (Biosource, Nivelles, Belgium). We determined fasting insulin, FSH, LH, total and free testosterone, sex hormone-binding globulin (SHBG) levels using ELISA kits (DRG International, USA). We calculated insulin resistance (IR) with the homeostatic model assessment-IR (HOMA-IR) index, which is defined as fasting serum insulin $(\mathrm{FSI})$ value $(\mu \mathrm{U} / \mathrm{ml}) \times$ fasting plasma glucose value $(\mathrm{mg} / \mathrm{dl}) / 405$. The B cell function was calculated using HOMA-B as $(\times 20$ (fasting insulin $\mu \mathrm{U} / \mathrm{mL}) /$ (fasting glucose $(\mathrm{mmol} / \mathrm{l})-3.5)[14]$.

\section{Determination of serum vitamin $D$ levels}

Serum concentrations of 25(OH)-D were tested using enzyme-linked immunosorbent assay, [Cat No. EQ 6411- 
9601, Euroimmun Medizinische Labordiagnostika AG, Germany]. Current recommendations define VD deficiency as serum 25(OH)-D levels less than $20 \mathrm{ng} / \mathrm{ml}$ and VD insufficiency less than $30 \mathrm{ng} / \mathrm{ml}$ [15].

\section{Carotid ultrasonography}

Carotid artery atherosclerosis was determined by one examiner for all patients across all six sites, using highresolution B-mode ultrasound $\left(\mathrm{M}\right.$-Turbo ${ }^{\circ}$, SonoSite, Washington, Bothell, USA), according to the American Society of Echocardiography protocol [16].

\section{Statistical analysis}

Data analyses were done with a statistical package for the social sciences software (SPSS Version 17, Chicago, Illinois). Data were expressed as mean \pm standard deviation (SD). The relationships of serum 25(OH)-D levels with clinical and laboratory parameters among PCOS patients were tested with the Pearson correlation. A stepwise multiple linear regression analysis was done to detect the main predictors of serum 25(OH)-D levels in the PCOS group. Logistic regression analysis was performed to determine the predictor biomarker associated with VD supplementation among PCOS patients. The appropriate sample size and power of the study were determined using PAWE-3D [17]. We considered $P$ to be significant at $<0.05$ with a $95 \%$ confidence interval $(\mathrm{CI})$.

\section{Results}

The final total numbers of PCOS women enrolled in this study were 95 , at the beginning of the study, 110 women with PCOS were included in the trial; 60 women with PCOS in the intervention group and 40 PCOS patients in the non-intervention group. Finally, 55 patients of the intervention group and 40 PCOS patients of the nonintervention group completed our study. Thus, we excluded all subjects who did not complete this study. Of note, there were no adverse effects of VD supplementation during the intervention.

\section{Clinical and biochemical characteristics of the studied groups}

In the PCOS group, we found significant higher levels of waist/hip ratio, systolic, and diastolic blood pressure, total cholesterol, triglycerides, LDL cholesterol, FPG, FSI, and HOMA-IR compared to the healthy control group. PCOS laboratory markers including total testosterone, free testosterone, androstenedione, DHEA-S, hirsutism score, ovarian volume, FSH, LH, LH/FSH, and antral follicle count (AFC) values were significantly high in PCOS cases compared with controls.

Additionally, the PCOS group had significantly higher levels of serum P, albumin, serum ALP, and CIMT compared to the control group. On the contrary, we detected significant lower values of serum 25(OH)-D, serum $\mathrm{Ca}$, SHBG, HDL, and HOMA-B levels in PCOS patients compared to the control group. Moreover, we observed significant higher levels of inflammatory markers; hsCRP, WBC, and neutrophil counts in PCOS cases compared to controls $\left(P^{>} 0.05\right)$, (Table 1$)$.

\section{Pearson correlation between serum 25(OH)-D (ng/ml) levels and clinical and laboratory parameters among PCOS women}

Serum $25(\mathrm{OH})$-D levels were negatively correlated with systolic blood pressure (Fig. 1), serum triglycerides (Fig. 2), HOMA-IR (Fig. 3), FSI (Fig. 4), and BMI (Fig. 5). Also, serum $25(\mathrm{OH})-\mathrm{D}$ concentrations were negatively correlated with CIMT ((Fig. 6), free testosterone (Fig. 7), AFC (Fig. 8), ovarian volume (Fig. 9), and hs-CRP (Fig. 10). However, the hirsutism score (Fig. 11) was non-significant correlated with serum 25(OH)-D levels.

\section{A stepwise multiple linear regression analysis in PCOS groups}

In PCOS group, stepwise multiple linear regression analysis revealed that CIMT, FSI, and FPG were the main predictors of serum 25(OH)-D levels among other clinical and laboratory biomarkers (Table 2).

The impact of vitamin D supplementations on clinicmetabolic status and androgenic profile among the studied group

In the non-intervention group $(n=40)$ after 12 weeks of trial, our results revealed a significant increase of BMI, TC, LDL, hs-CRP, WBC, and neutrophil counts, whereas HDL level was significantly decreased.

Considering the impact of VD supplementation in the intervention group (42,000 IU oral vitamin D per week and $500 \mathrm{mg}$ calcium carbonate per day for 12 weeks), on body composition parameters, BMI and WHR were significantly decreased. Moreover, cardio-metabolic risks were improved after VD supplementations (Table 3).

Regarding the PCOS phenotype, androgenic profile, and inflammatory markers, there were significantly decreased values after the intervention. Even more important, 25(OH)-D serum levels were increased after VD supplementations (Table 3).

\section{Assessment of the power of VD supplementation to improve cardio-metabolic risk and androgenic markers of women with PCOS}

Logistic regression analysis was performed to detect the main predictors of cardio-metabolic biomarkers associated with VD supplementation among PCOS patients. Our findings revealed that among clinical and laboratory features of PCOS, androstenedione, FSI, and hirsutism score were independent predictors of improvement of VD supplementation 
Table 1 Clinical and biochemical characteristics of the studied groups

\begin{tabular}{|c|c|c|c|}
\hline & $\begin{array}{l}\text { Control group } \\
\text { (mean } \pm \text { SD) } \\
(n=50)\end{array}$ & $\begin{array}{l}\text { PCO patients } \\
\text { (mean } \pm \text { SD) } \\
(n=95)\end{array}$ & $P$ \\
\hline Age (years) & $27.98 \pm 6.11$ & $26.05 \pm 3.72$ & 0.085 \\
\hline $\mathrm{BMI}(\mathrm{kg} / \mathrm{m} 2)$ & $26.79 \pm 2.84$ & $27.99 \pm 5.772$ & 0.564 \\
\hline Waist/hip ratio & $0.91 \pm 0.103$ & $1.49 \pm 0.321$ & $<0.001^{*}$ \\
\hline Systolic blood pressure (mmHg) & $117.96 \pm 6.85$ & $125.9 \pm 5.89$ & $<0.001^{*}$ \\
\hline Diastolic blood pressure $(\mathrm{mmHg})$ & $72.74 \pm 8.05$ & $89.18 \pm 3.82$ & $<0.001^{*}$ \\
\hline Hirsutism score & $5.54 \pm 0.54$ & $8.35 \pm 5.81$ & $<0.001^{*}$ \\
\hline Ovarian volume & $7.43 \pm 1.41$ & $15.41 \pm 4.147$ & $<0.001^{*}$ \\
\hline AFC & $5.42 \pm 0.92$ & $14.91 \pm 5.147$ & $<0.001^{*}$ \\
\hline T.cholesterol (mg/dL) & $158.8 \pm 14.6$ & $202.35 \pm 31.73$ & $<0.001^{*}$ \\
\hline Triglycerides (mg/dL) & $143.2 \pm 23.04$ & $194.8 \pm 46.02$ & $<0.001^{*}$ \\
\hline LDL.C(mg/dL) & $76.5 \pm 13.84$ & $120.39 \pm 31.04$ & $<0.001^{*}$ \\
\hline HDL.C(mg/dL) & $53.7 \pm 3.26$ & $42.9 \pm 6.22$ & $<0.001^{*}$ \\
\hline $\mathrm{FPG}(\mathrm{mg} / \mathrm{dL})$ & $85.2 \pm 8.02$ & $87.96 \pm 3.5$ & $<0.001^{*}$ \\
\hline $\mathrm{FSI}(\mu \mathrm{U} / \mathrm{mL})$ & $6.2 \pm 1.75$ & $11.26 \pm 6.2$ & $<0.001^{*}$ \\
\hline HOMA-IR & $1.2 \pm 0.23$ & $2.45 \pm 1.36$ & $<0.001^{*}$ \\
\hline HOMA $\beta$ & $172.2 \pm 117.6$ & $199.19 \pm 57.57$ & $<0.001^{*}$ \\
\hline Total testosterone $(\mathrm{ng} / \mathrm{mL})$ & $0.56 \pm 0.16$ & $0.784 \pm 0.36$ & $<0.001^{*}$ \\
\hline Free testosterone $(\mathrm{pg} / \mathrm{mL})$ & $0.998 \pm 0.347$ & $2.43 \pm 1.21$ & $<0.001^{*}$ \\
\hline SHBG (nmol/L) & $88.95 \pm 11.9$ & $36.89 \pm 18.63$ & $<0.001^{*}$ \\
\hline DHEA-S $(\mu \mathrm{g} / \mathrm{dl}))$ & $162.44 \pm 26.54$ & $212.45 \pm 81.15$ & $<0.001^{*}$ \\
\hline Androstenedione (ng/mL) & $1.19 \pm 0.36$ & $2.86 \pm 1.93$ & $<0.001^{*}$ \\
\hline $\mathrm{FSH}(\mathrm{mlU} / \mathrm{mL})$ & $6.84 \pm 2.044$ & $8.59 \pm 0.467$ & $<0.001^{*}$ \\
\hline $\mathrm{LH}(\mathrm{mlU} / \mathrm{mL})$ & $8.21 \pm 0.54$ & $10.57 \pm 2.41$ & $<0.001^{*}$ \\
\hline $\mathrm{LH} / \mathrm{FSH}$ & $1.06 \pm 0.15$ & $1.14 \pm 0.122$ & $<0.001^{*}$ \\
\hline 25(OH)-D (ng/ml) & $39.54 \pm 8.13$ & $14.27 \pm 4.48$ & $<0.001^{*}$ \\
\hline $\mathrm{Ca}(\mathrm{mg} / \mathrm{dl})$ & $9.20 \pm 0.97$ & $8.22 \pm 0.89$ & $<0.001^{*}$ \\
\hline $\mathrm{PO} 4$ (mg/dl) & $4.29 \pm 0.83$ & $5.48 \pm 0.597$ & $<0.001^{*}$ \\
\hline Albumin (g/dl) & $3.99 \pm 0.15$ & $4.26 \pm 0.637$ & $<0.001^{*}$ \\
\hline Alkaline phosphatase (IU/L) & $105.6 \pm 17.3$ & $127.67 \pm 30.3$ & $<0.001^{*}$ \\
\hline WBC count $($ cell $\times 103 / \mu l)$ & $4.96 \pm 1.83$ & $7.94 \pm 1.766$ & $<0.001^{*}$ \\
\hline Neutrophil count $\left(\right.$ cell $\left.\times 10^{3} / \mu \mathrm{l}\right)$ & $2.71 \pm 0.66$ & $3.79 \pm 1.09$ & $<0.001^{*}$ \\
\hline hs-CRP ( $\mu \mathrm{g} / \mathrm{ml})$ & $2.29 \pm 0.38$ & $4.976 \pm 0.952$ & $<0.001^{*}$ \\
\hline $\mathrm{CIMT}(\mathrm{mm})$ & $0.77 \pm 0.186$ & $1.44 \pm 0.22$ & $<0.001^{*}$ \\
\hline
\end{tabular}

$B M I$ body mass index, $A F C$ antral follicle count, $F S I$ fasting serum insulin, $F P G$ fasting plasma glucose, HOMA-IR homeostasis model assessments of insulin resistance, SHBG sex hormone-binding globulin, DHEA-S dehydroepiandrosteron sulfate, ALP alkaline phosphatase, hs-CRP high-sensitivity C-reactive protein, CIMT carotid intima-media thickness

${ }^{*} P<0.05$ when compared with control group

with odds ratios of $0.253,1.199$, and 1.330 , respectively (Table 4).

\section{Discussion}

As a matter of fact, PCOS is the most common ovarian disorder that affects $6-10 \%$ women worldwide [1] and accounts for $70 \%$ of cases of anovulatory infertility. PCOS is a set of reproductive and metabolic symptoms associated with an imbalance of reproductive hormones in women [2].

The pandemic of VD deficiency represents a major public health concern, as this disorder is associated with an increased risk of medical comorbidities contributing to a significant rise in mortality. There are intriguing reports suggesting that VD deficiency is more prevalent among women with PCOS [18]. Few studies explored 


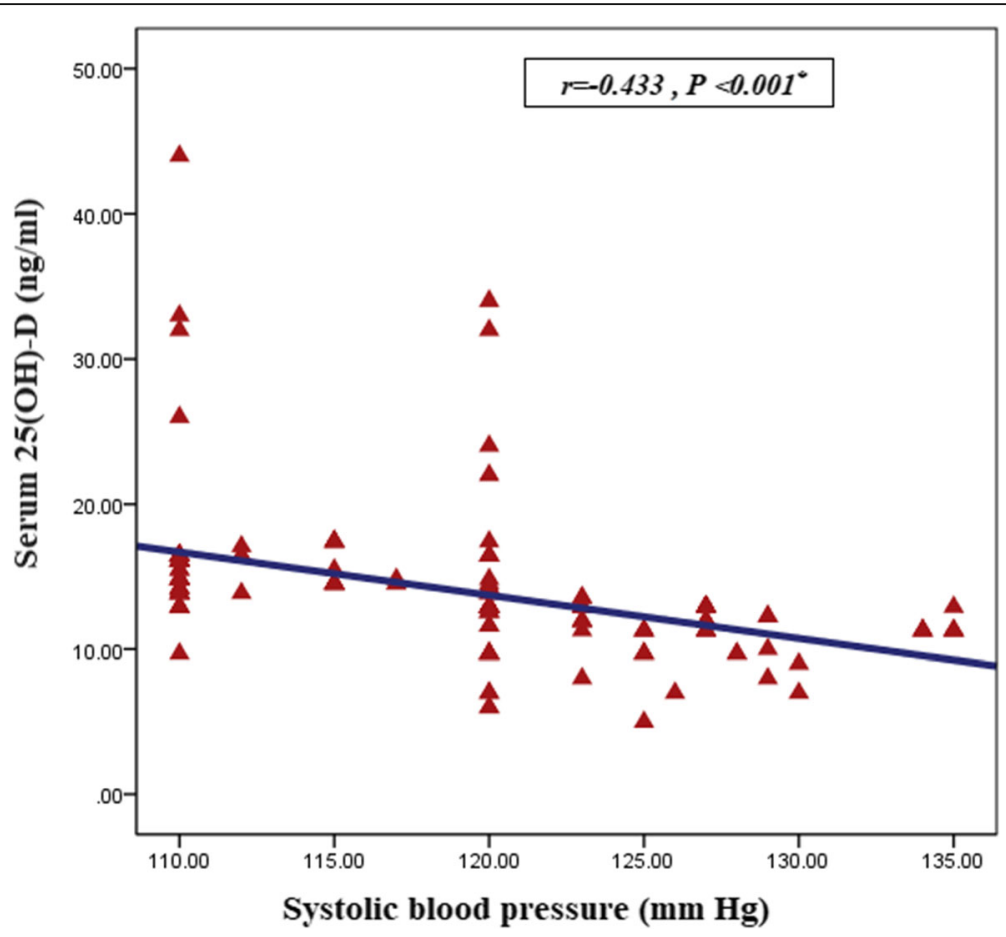

Fig. 1 Correlation between serum 25(OH)-D and systolic blood pressure

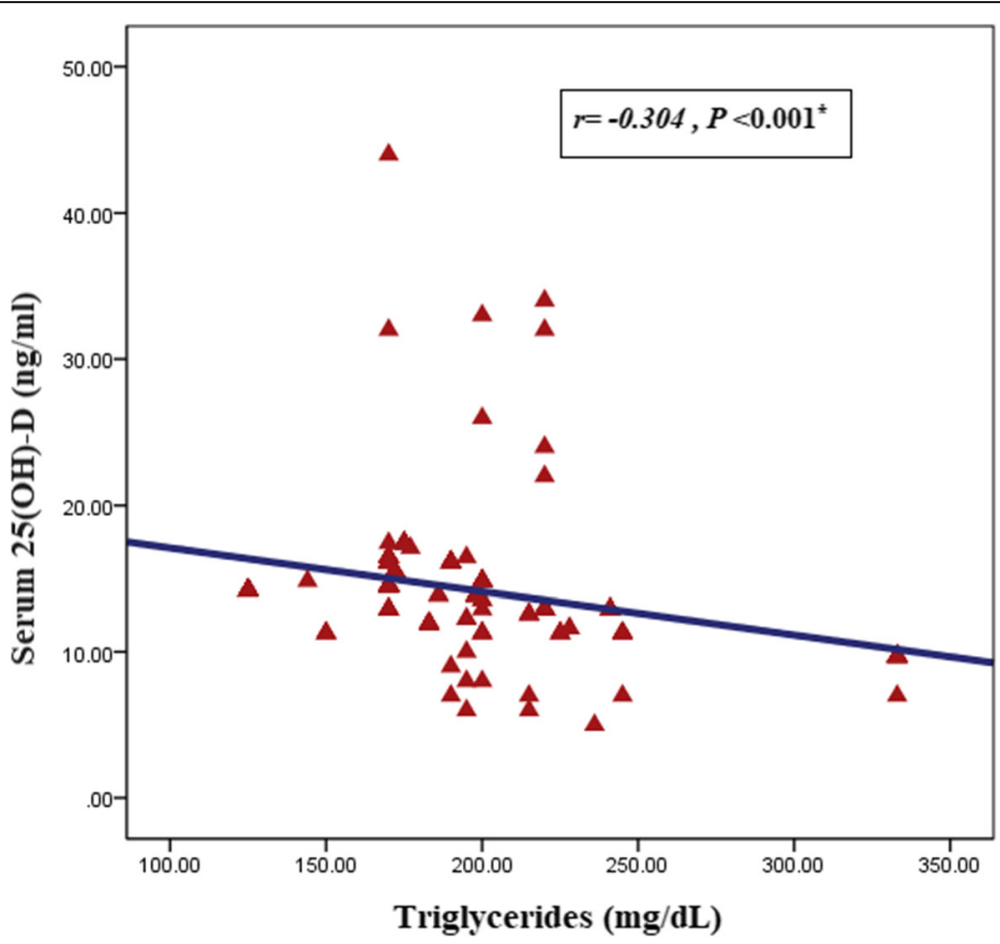

Fig. 2 Correlation between serum 25(OH)-D and serum triglycerides 


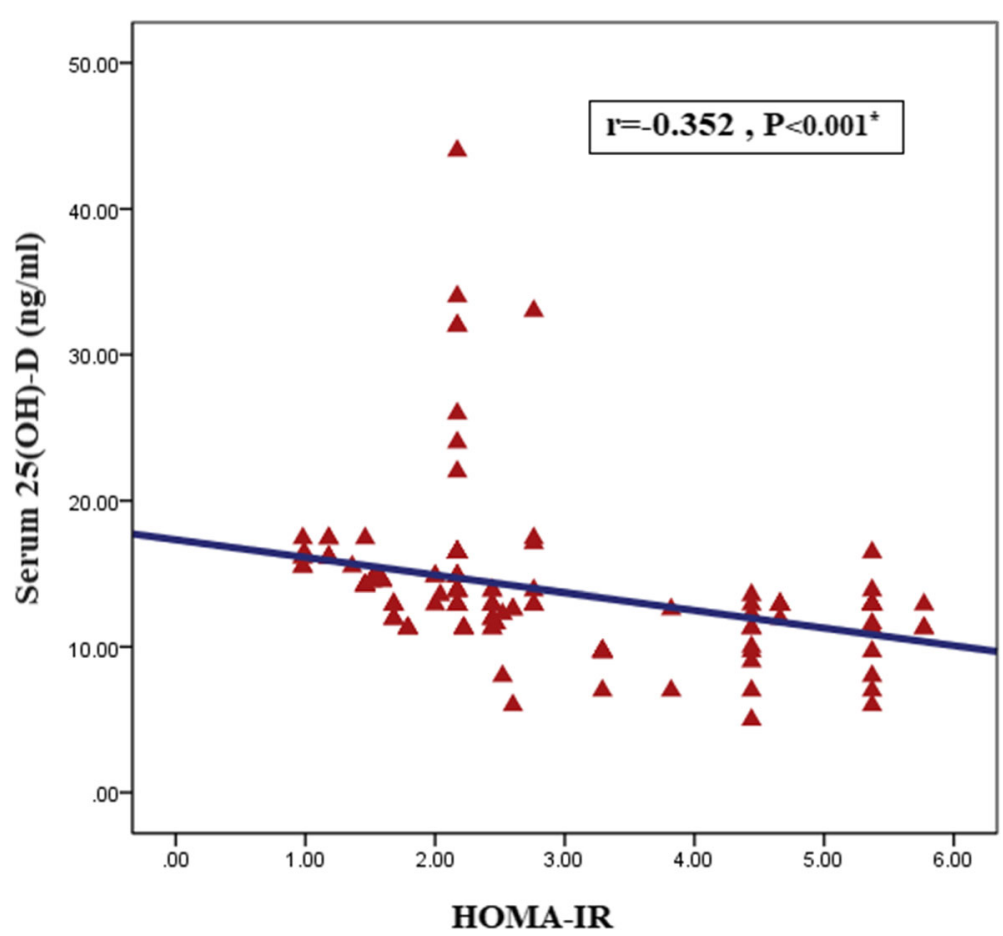

Fig. 3 Correlation between serum 25(OH)-D and HOMA-IR

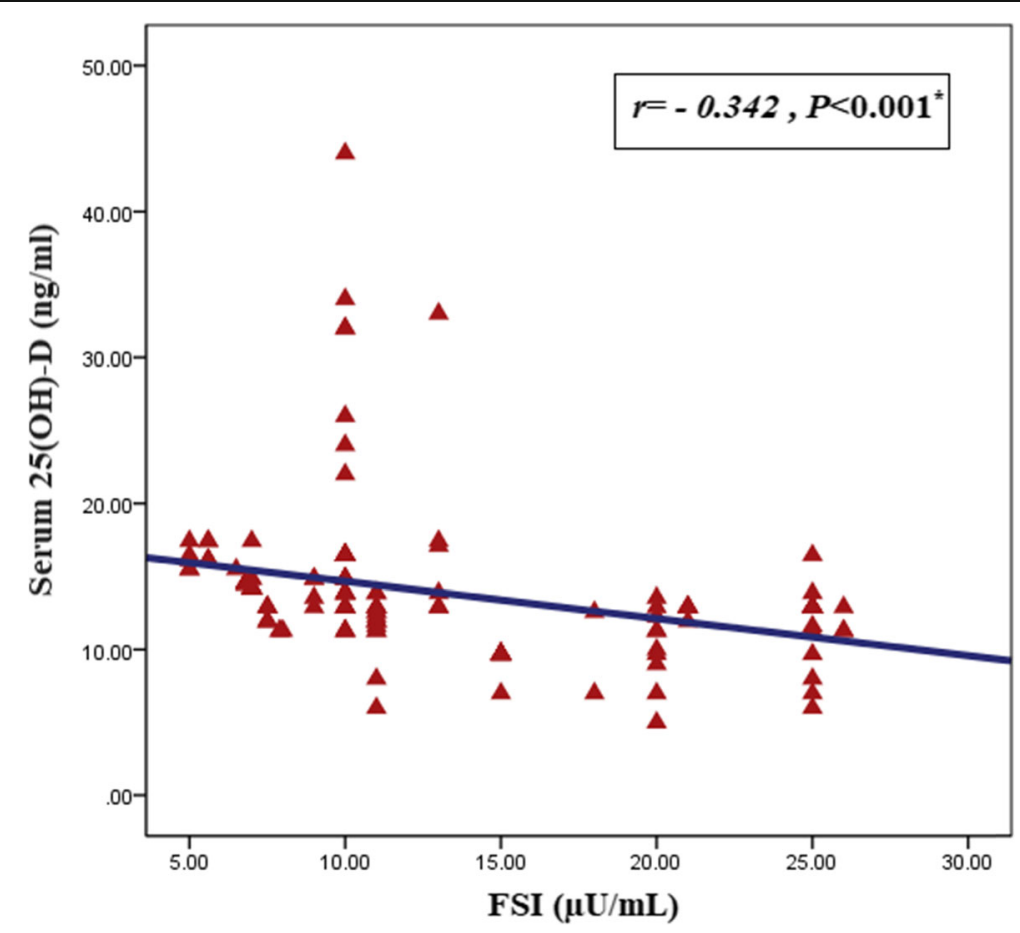

Fig. 4 Correlation between serum 25(OH)-D and fasting serum insulin 


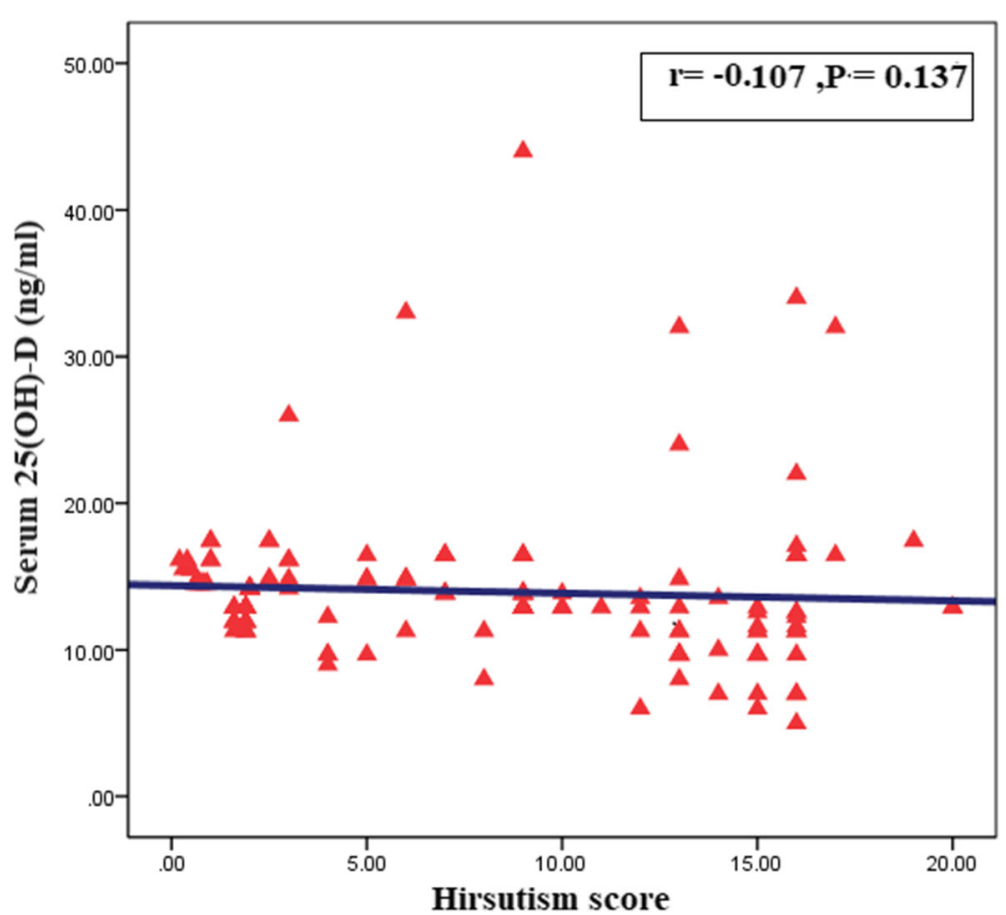

Fig. 5 Correlation between serum 25(OH)-D and hirsutism score

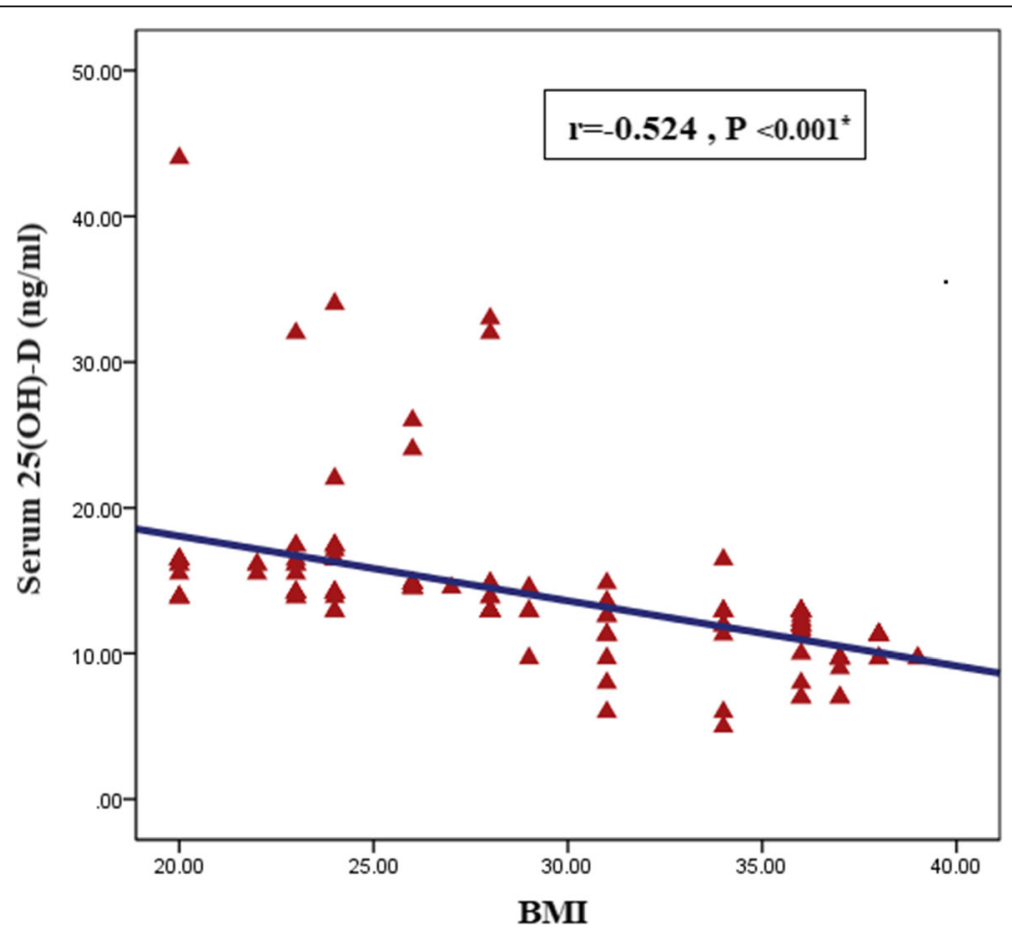

Fig. 6 Correlation between serum 25(OH)-D and BMI 


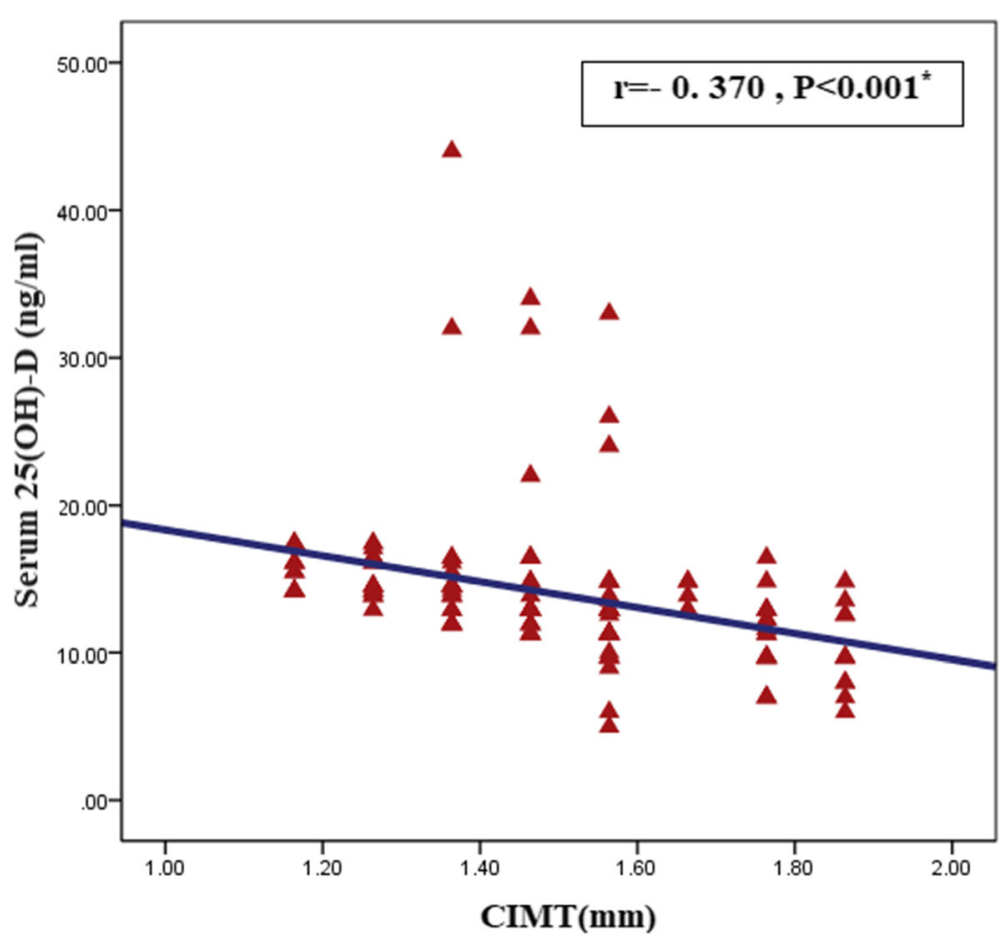

Fig. 7 Correlation between serum 25(OH)-D and CIMT

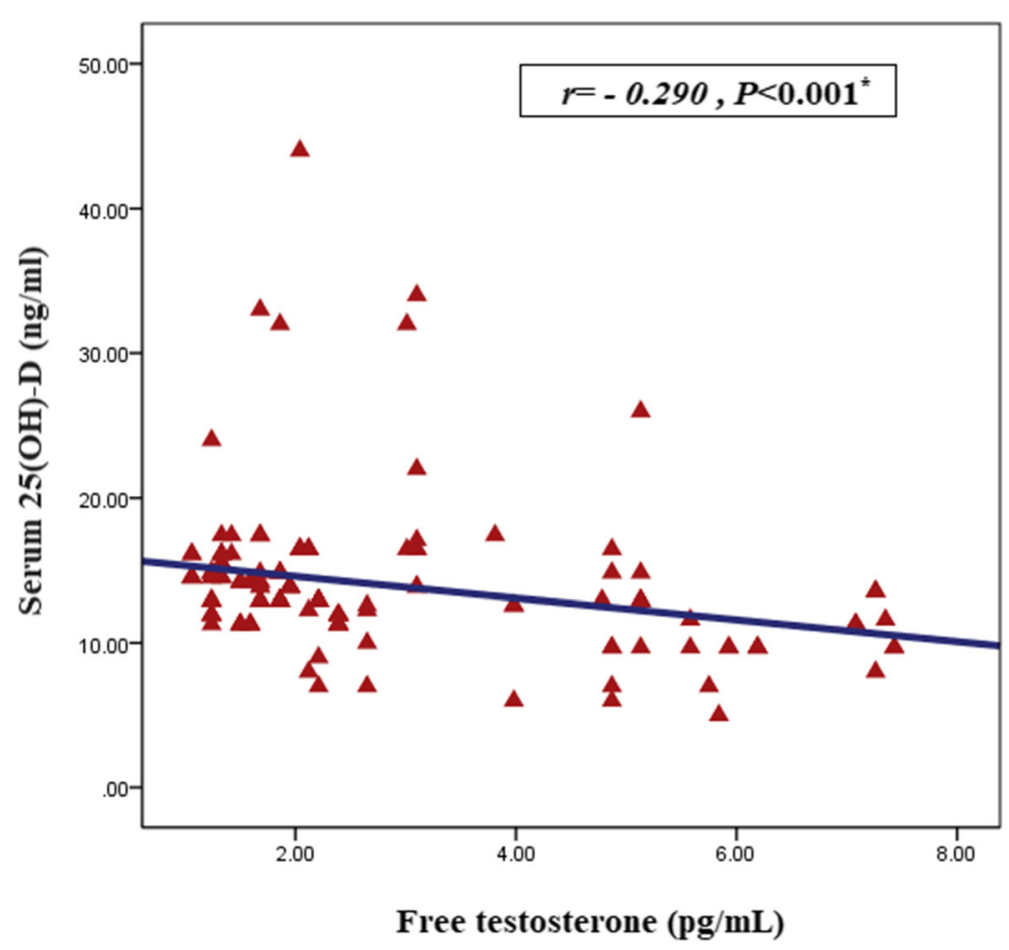

Fig. 8 Correlation between serum 25(OH)-D and free testosterone 


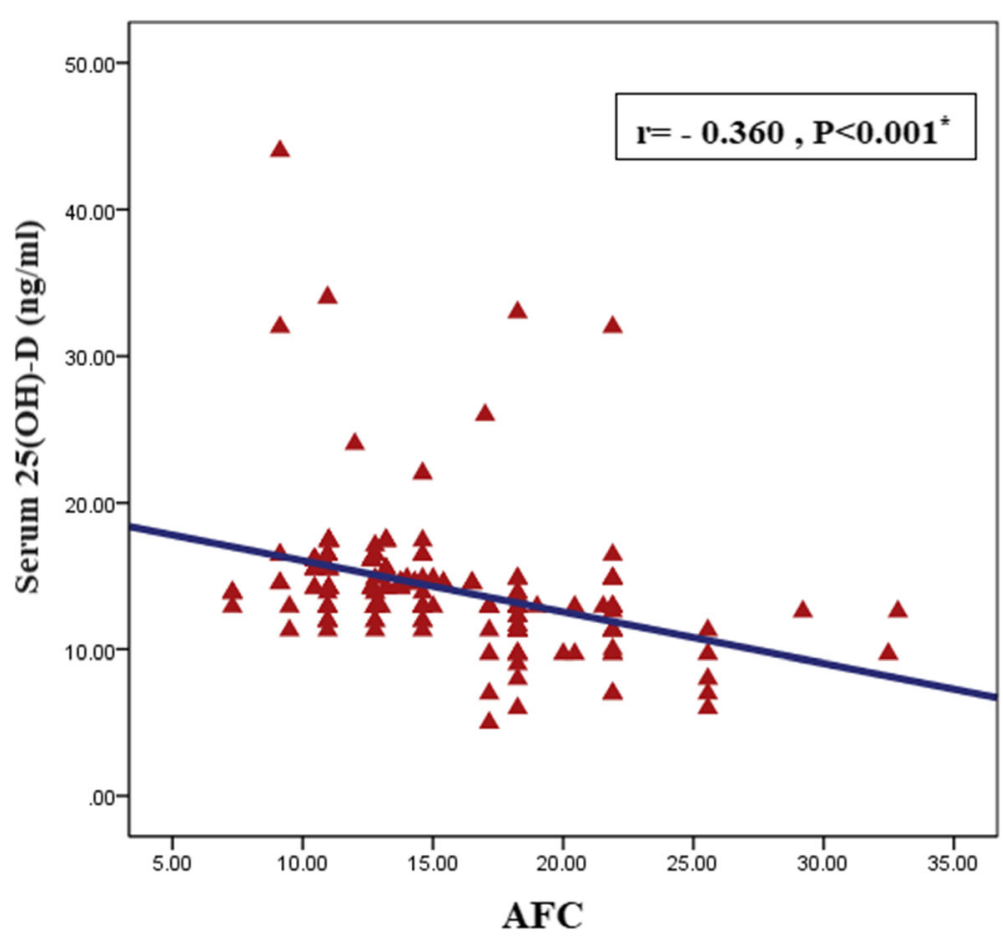

Fig. 9 Correlation between serum 25(OH)-D and AFC

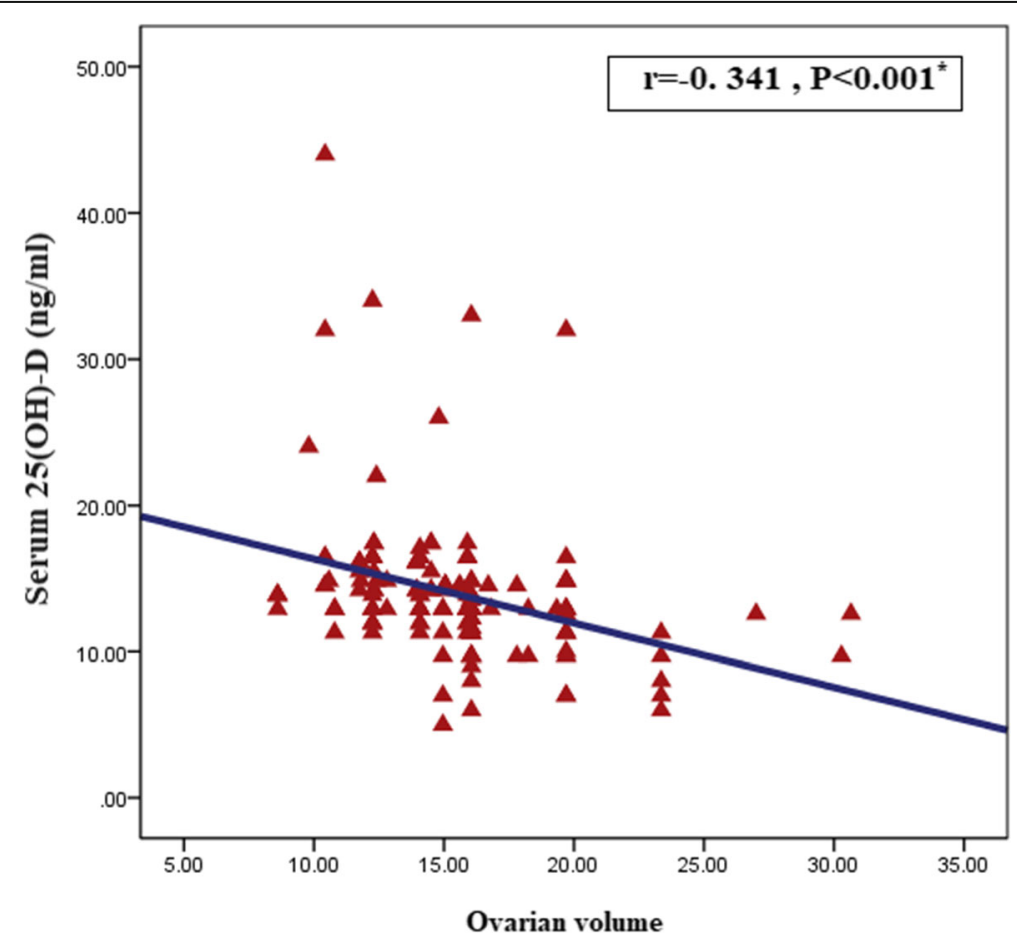

Fig. 10 Correlation between serum 25(OH)-D and ovarian volume 


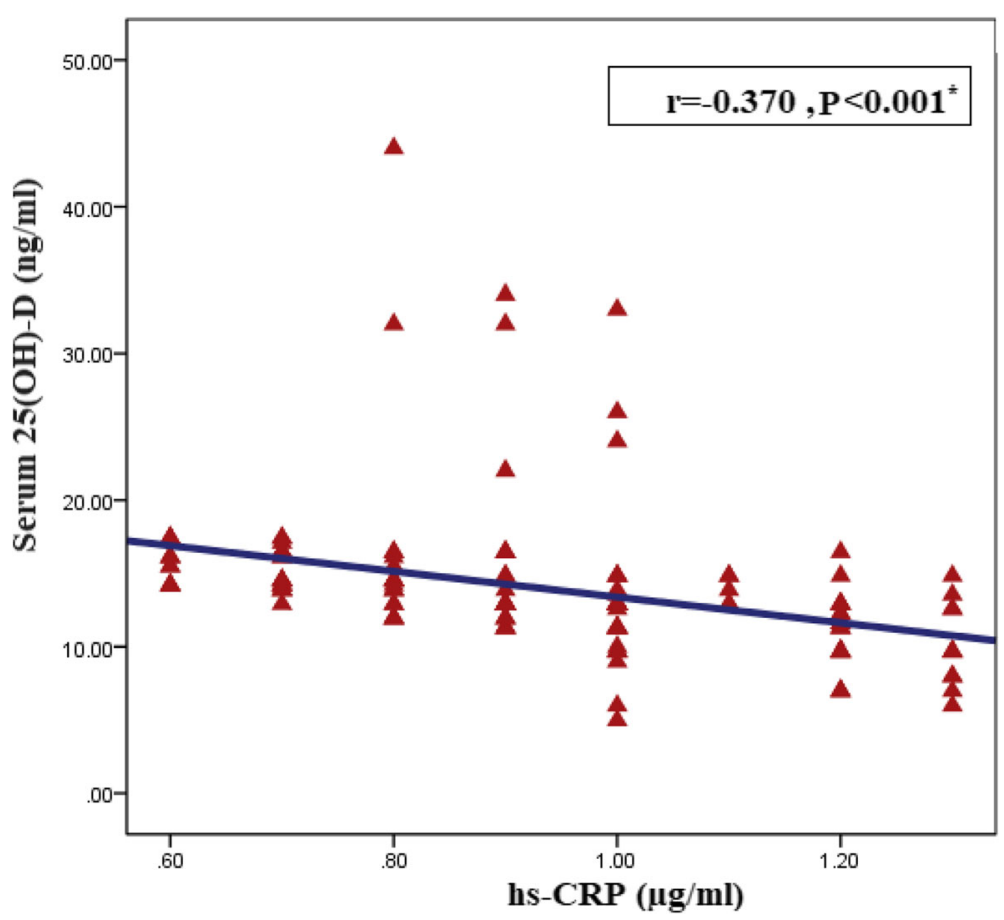

Fig. 11 Correlation between serum 25(OH)-D and hs-CRP

the association between VD deficiency with hirsutism score, IR, and obesity in PCOS [19]. Because the current treatment of PCOS is still suboptimal, and cardiometabolic diseases are the common cause of death all over the world, we aimed in the present study to investigate the levels of 25(OH)-D in PCOS patients. We also aimed to clarify the impact of vitamin D supplementation cardio-metabolic status, androgen profile, and clinical features of PCOS.

In order to assess the risk of cardiovascular disease among PCOS, our results revealed that the cardiometabolic factors; systolic blood pressure, TC, TG, LDL cholesterol, FPG, 2-h plasma glucose, FSI, HOMA-IR, and CIMT were significantly high in PCOS compared to control group. Our previous experience also suggested that PCOS women had higher values of parameters of hyperglycemia and dyslipidemia as well as obesity indices [20-22].

Our findings are in concordance with Glueck et al. who found that PCOS patients are at an increased risk of metabolic syndrome and cardiovascular risk factors in later life [23].

Supporting our results, Zhao et al. detected a high risk of coronary heart disease in PCOS [24]. Moreover,

Table 2 Multiple stepwise linear regression analyses in PCOS women to test the influence of the main independent variables against serum 25(OH)-D (ng/ml) levels (dependent variable) in PCOS women

\begin{tabular}{|c|c|c|c|c|c|c|c|c|}
\hline \multicolumn{2}{|c|}{ Model } & \multicolumn{2}{|c|}{ Unstandardized coefficients } & \multirow{2}{*}{$\begin{array}{l}\text { Standardized coefficients } \\
\beta\end{array}$} & \multirow[t]{2}{*}{$t$} & \multirow[t]{2}{*}{$P$ value } & \multicolumn{2}{|l|}{ 95\% C.I. } \\
\hline & & $\beta$ & SE & & & & Lower bound & Upper bound \\
\hline \multirow[t]{2}{*}{1} & Constant & 32.628 & 4.418 & & 7.385 & $<0.001^{*}$ & 23.871 & 41.386 \\
\hline & $\mathrm{CIMT}(\mathrm{mm})$ & -12.036 & 2.805 & -0.382 & -4.291 & $<0.001^{*}$ & -17.595 & -6.476 \\
\hline \multirow[t]{3}{*}{2} & Constant & 32.215 & 4.326 & & 7.446 & $<0.001^{*}$ & 23.638 & 40.791 \\
\hline & $\mathrm{CIMT}(\mathrm{mm})$ & -9.845 & 2.891 & -0.313 & -3.406 & $<0.001^{*}$ & -15.576 & -4.114 \\
\hline & $\mathrm{FSI}(\mu \mathrm{U} / \mathrm{mL})$ & -0.209 & 0.087 & -0.221 & -2.410 & $<0.05^{*}$ & -0.382 & -0.037 \\
\hline \multirow[t]{4}{*}{3} & Constant & 93.130 & 20.671 & & 4.505 & $<0.001^{*}$ & 52.148 & 134.112 \\
\hline & $\mathrm{CIMT}(\mathrm{mm})$ & -7.383 & 2.905 & -0.234 & -2.541 & $<0.05^{*}$ & -13.143 & -1.623 \\
\hline & $\mathrm{FSI}(\mu \mathrm{U} / \mathrm{mL})$ & -0.285 & 0.087 & -0.301 & -3.258 & $<0.001^{*}$ & 0.458 & -0.112 \\
\hline & $\mathrm{FPG}(\mathrm{mg} / \mathrm{dL})$ & -0.717 & 0.238 & -0.269 & -3.009 & $<0.001^{*}$ & -1.189 & -0.244 \\
\hline
\end{tabular}

*Statistically significant $(P<0.05)$ 
Table 3 The impact of vitamin D supplementations on cardio-metabolic status and androgenic profile in PCOS group

\begin{tabular}{|c|c|c|c|c|c|c|}
\hline & \multicolumn{3}{|c|}{ Non-intervention group $(n=40)$} & \multicolumn{3}{|c|}{ Intervention group $(n=55)$} \\
\hline & Before & After & $P$ value & Before & After & $P$ value \\
\hline $\mathrm{BMI}\left(\mathrm{kg} / \mathrm{m}^{2}\right)$ & $23.72 \pm 2.34$ & $26.37 \pm 4.84$ & $<0.001^{*}$ & $33.43 \pm 3.96$ & $26.8 \pm 5.74$ & $<0.001^{*}$ \\
\hline Waist/hip ratio & $1.07 \pm .196$ & $1.124 \pm 0.19$ & 0.341 & $1.491 \pm .32113$ & $1.147 \pm 0.180$ & $<0.001^{*}$ \\
\hline $\mathrm{SBP}(\mathrm{mmHg})$ & $113.8 \pm 3.59$ & $116.4 \pm 5.26$ & 0.050 & $123.1 \pm 6.6$ & $116.8 \pm 7.02$ & $<0.001^{*}$ \\
\hline $\mathrm{DBP}(\mathrm{mmHg})$ & $71.05 \pm 8.23$ & $72.72 \pm 7.29$ & 0.314 & $77.47 \pm 7.50$ & $69.27 \pm 6.77$ & $<0.001^{*}$ \\
\hline Hirsutism score & $6.35 \pm 0.90$ & $6.50 \pm 0.78$ & 0.839 & $11.83 \pm 3.86$ & $9.4 \pm 4.50$ & $<0.001^{*}$ \\
\hline Ovarian volume & $13.61 \pm 1.80$ & $13.99 \pm 1.72$ & 0.536 & $18.31 \pm 3.697$ & $13.58 \pm 2.80$ & $<0.001^{*}$ \\
\hline AFC & $12.31 \pm 1.80$ & $12.69 \pm 1.72$ & 0.575 & $20.5 \pm 3.69$ & $13.36 \pm 3.68$ & $<0.001^{*}$ \\
\hline T. cholesterol (mg/dL) & $175.5 \pm 11.4$ & $186.9 \pm 22.66$ & $<0.05^{*}$ & $232.3 \pm 20.9$ & $203.2 \pm 31.6$ & $<0.001^{*}$ \\
\hline Triglycerides (mg/dL) & $164.4 \pm 20.9$ & $168.6 \pm 27.3$ & 0.617 & $232.3 \pm 57.3$ & $198.6 \pm 25.01$ & $<0.001^{*}$ \\
\hline $\mathrm{LDL} . C(\mathrm{mg} / \mathrm{dL})$ & $94.86 \pm 17.1$ & $108.1 \pm 25.8$ & $<0.05^{*}$ & $148.7 \pm 16.1$ & $119.5 \pm 31.63$ & $<0.001^{*}$ \\
\hline $\mathrm{HDL} . C(\mathrm{mg} / \mathrm{dL})$ & $47.5 \pm 3.36$ & $45.12 \pm 4.97$ & $<0.05^{*}$ & $37.14 \pm 5.18$ & $44.01 \pm 5.338$ & $<0.001^{*}$ \\
\hline FPG (mg/dL) & $86.05 \pm 4.77$ & $87.47 \pm 4.21$ & 0.057 & $89.38 \pm 2.38$ & $88.29 \pm 1.74$ & $<0.001^{*}$ \\
\hline $\mathrm{FSI}(\mu \mathrm{U} / \mathrm{mL})$ & $6.1 \pm 0.83$ & $7.74 \pm 4.02$ & 0.140 & $16.5 \pm 6.108$ & $12.28 \pm 5.251$ & $<0.001^{*}$ \\
\hline HOMA-IR & $1.31 \pm 0.23$ & $1.67 \pm 0.91$ & 0.111 & $3.61 \pm 1.287$ & $2.67 \pm 1.15$ & $<0.001^{*}$ \\
\hline HOMA- $\beta$ & $98.2 \pm 13.1$ & $113.5 \pm 50.72$ & 0.332 & $231.3 \pm 98.1$ & $174.8 \pm 73.6$ & $<0.001^{*}$ \\
\hline T.testosterone $(\mathrm{ng} / \mathrm{mL})$ & $0.605 \pm 0.33$ & $0.62 \pm 0.29$ & 0.789 & $0.93 \pm 0.35$ & $0.88 \pm 0.34$ & $<0.001^{*}$ \\
\hline SHBG (nmol/L) & $48.8 \pm 17.1$ & $45.65 \pm 18.52$ & 0.390 & $26.5 \pm 17.14$ & $32.25 \pm 13.07$ & 0.067 \\
\hline DHEA-S $(\mu \mathrm{g} / \mathrm{dl}))$ & $171.2 \pm 49.6$ & $169.4 \pm 48.6$ & 0.908 & $276.4 \pm 79.77$ & $209.7 \pm 80.51$ & $<0.001^{*}$ \\
\hline Androstenedione $(\mathrm{ng} / \mathrm{mL})$ & $3.3 \pm 1.94$ & $3.55 \pm 1.83$ & 0.626 & $3.45 \pm 2.196$ & $1.42 \pm 0.404$ & 0.440 \\
\hline $\mathrm{FSH}(\mathrm{mlU} / \mathrm{mL})$ & $9.1 \pm 0.15$ & $8.96 \pm 0.156$ & 0.451 & $8.54 \pm 0.27$ & $8.08 \pm 0.403$ & $<0.001^{*}$ \\
\hline $\mathrm{LH}(\mathrm{mlU} / \mathrm{mL})$ & $9.6 \pm 1.4$ & $9.6 \pm 1.19$ & 0.954 & $12.8 \pm 3.153$ & $9.69 \pm 0.66$ & $<0.001^{*}$ \\
\hline $\mathrm{LH} / \mathrm{FSH}$ & $1.07 \pm 0.16$ & $1.07 \pm 0.13$ & 0.965 & $1.13 \pm 0.098$ & $1.6 \pm 0.42$ & $<0.001^{*}$ \\
\hline $25(\mathrm{OH})-\mathrm{D}(\mathrm{ng} / \mathrm{ml})$ & $15.32 \pm 1.085$ & $14.55 \pm 1.60$ & 0.383 & $10.9 \pm 2.469$ & $26.6 \pm 6.64$ & $<0.001^{*}$ \\
\hline $\mathrm{Ca}(\mathrm{mg} / \mathrm{dl})$ & $8.14 \pm 0.83$ & $8.31 \pm 0.831$ & 0.404 & $8.17 \pm 0.87$ & $8.26 \pm 1.01$ & 0.607 \\
\hline PO4 (mg/dl) $(\mathrm{mg} / \mathrm{dl}$ & $5.43 \pm 0.5$ & $5.54 \pm 0.55$ & 0.404 & $5.44 \pm 0.58$ & $5.5 \pm .674$ & 0.883 \\
\hline ALP $(U / L)$ & $100.7 \pm 13.5$ & $106.5 \pm 16.26$ & 0.142 & $163.9 \pm 18.5$ & $127.1 \pm 19.9$ & $<0.001^{*}$ \\
\hline WBC count $($ cell $\times 103 / \mu l)$ & $5.03 \pm 0.934$ & $5.65 \pm 1.45$ & $<0.001^{*}$ & $8.70 \pm 0.66$ & $7.50 \pm 0.945$ & $<0.001^{*}$ \\
\hline Neutrophil count $\left(\right.$ cell $\left.\times 10^{3} / \mu \mathrm{l}\right)$ & $2.71 \pm 0.668$ & $3.1 \pm 0.9$ & $<0.001^{*}$ & $4.72 \pm 0.661$ & $4.17 \pm 0.702$ & $<0.001^{*}$ \\
\hline hs-CRP ( $\mu \mathrm{g} / \mathrm{ml})$ & $4.6 \pm 0.668$ & $4.9 \pm 0.97$ & $<0.001^{*}$ & $6.6 \pm 0.661$ & $6.0 \pm 0.70$ & $<0.001^{*}$ \\
\hline $\mathrm{CIMT}(\mathrm{mm})$ & $1.25 \pm 0.093$ & $1.29 \pm 0.112$ & 0.142 & $1.689 \pm 0.127$ & $1.440 \pm 0.13$ & $<0.001^{*}$ \\
\hline
\end{tabular}

*Statistically significant $(P<0.05)$

Morgan et al. study detected higher mortality in PCOS compared to the control group [25].

Regarding lipid profile in PCOS women, similar to our results Wild et al. showed that women with PCOS have higher levels of TG, LDL cholesterol, and TC, and lower HDL cholesterol levels compared with the control group [26]. In addition, Mulhim et al. conducted their study to assess the metabolic and clinical profile of Saudi patients with PCOS, they found that PCOS patients had non- significantly difference in TC, HDL cholesterol, TG, LDL cholesterol, or FPG values compared to control group [27].

In order to better elucidate the levels of inflammatory markers in PCOS. In our research, we found significant higher levels of inflammatory markers; WBC, neutrophil as well as hs-CRP in PCOS cases compared to controls.

Similar to these results, our previous finding showed that PCOS patients had significant higher values of inflammatory biomarkers including serum procalcitonin, hs-CRP, WBC, and neutrophil counts compared to healthy women [20-22]. Altogether, these data point to VD acts to lower systemic inflammation in general by interacting with transcription and expression of cytokine genes [28-30].

Omics studies have indeed demonstrated that VD could under-regulate the activation of nuclear factor- $\mathrm{k} B$ [30], which plays a regulatory role for pro-inflammatory cytokine generation associated with the resistance of insulin $[31,32]$. 
Table 4 logistic regression analysis evaluating the association of cardio-metabolic risk and androgenic markers with VD supplementation among PCOS women

\begin{tabular}{|c|c|c|c|c|c|c|c|}
\hline & \multicolumn{2}{|c|}{ Unstandardized coefficients } & \multirow{2}{*}{$\begin{array}{l}\text { Odds } \\
\text { ratio }\end{array}$} & \multicolumn{2}{|l|}{ 95\% C.I. } & \multirow[t]{2}{*}{$t$} & \multirow{2}{*}{$\begin{array}{l}P \\
\text { value }\end{array}$} \\
\hline & $\bar{\beta}$ & Standard error & & Lower bound & Upper bound & & \\
\hline Constant & -68.191 & 47.583 & 0.000 & & 2.035 & 2.054 & 0.152 \\
\hline SHBG & 0.001 & 0.032 & 1.001 & 0.941 & 1.065 & 0.001 & 0.973 \\
\hline Androstenedione & -1.374 & 0.485 & 0.253 & 0.098 & 0.654 & 8.038 & $<0.05^{*}$ \\
\hline Free testosterone & 0.104 & 0.369 & 1.109 & 0.539 & 2.284 & 0.079 & 0.779 \\
\hline Systolic BP & -0.092 & 0.158 & 0.912 & 0.669 & 1.243 & 0.342 & 0.559 \\
\hline Diastolic BP & 0.164 & 0.152 & 1.179 & 0.876 & 1.587 & 1.175 & 0.278 \\
\hline Total cholesterol & -0.030 & 0.119 & 0.971 & 0.769 & 1.224 & 0.064 & 0.801 \\
\hline LDL cholesterol & 0.029 & 0.123 & 1.030 & 0.809 & 1.311 & 0.057 & 0.811 \\
\hline HDL cholesterol & -0.129 & 0.153 & 0.879 & 0.651 & 1.187 & 0.708 & 0.400 \\
\hline FPG & 0.764 & 0.504 & 2.146 & 0.799 & 5.764 & 2.294 & 0.130 \\
\hline FSI & 0.182 & 0.091 & 1.199 & 1.004 & 1.433 & 4.017 & $<0.05^{*}$ \\
\hline Hirsutism score & 0.285 & 0.156 & 1.330 & 0.981 & 1.804 & 3.366 & $<0.05^{*}$ \\
\hline
\end{tabular}

*Statistically significant $(P<0.05)$

We in this study attempted to explore the levels of serum 25(OH)-D in PCOS, we found significant lower levels of serum 25(OH)-D in PCOS women compared with healthy controls. Even more importantly, our results reported that $25(\mathrm{OH})$-D levels were negatively correlated with cardio-metabolic risk including, systolic blood pressure, serum triglycerides, HOMA-IR, FSI, BMI, CIMT, free testosterone, AFC, ovarian volume, and hs-CRP.

Our findings are in concordance with Wehr et al. hypovitaminosis D which was common in PCOS women [33]. Similar results were described in Hahn et al. study which was conducted on women with PCOS, and they found lower levels of serum 25(OH)-D in PCOS women [34].

In that context, a study conducted by Jia et al. observed that the serum concentration of vitamin D is associated with the risk of PCOS. Nonetheless, they did not observe any improvement of clinical and laboratory features after vitamin D3 supplementation for women with PCOS [35].

In contrast, a study by Panidis et al. [36] found no significant difference of serum 25(OH)-D concentrations between women with PCOS and controls.

Supporting our results, $\mathrm{Li}$ et al. found that metabolic risk factors were associated with lower levels of serum 25(OH)-D in PCOS women [37]. Additionally, Wehr et al. [33] observed that the serum 25OH-D level is positively correlated with insulin sensitivity and negatively correlated with IR in women with PCOS.

In earlier published studies conducted in our region, Middle East [38], Zhang et al. observed low VD in diabetes and the possible mechanisms for this association may include the presence of vitamin D receptors in pancreatic beta cells to which circulating vitamin D binds [39]. In addition, VD has been well recognized for its role in regulating extracellular calcium flux, and insulin secretion is known as a calcium-dependent process [40].

Similar to our results, some studies report inverse associations between serum 25(OH)-D levels and testosterone among women with PCOS [33, 41-43].

Our finding adds to the growing body of evidence implicating that women with PCOS tend to have decreased vitamin D levels, and vitamin D deficiency has been correlated with increased IR, BMI, total testosterone, and DHEA-S [34].

There is scattered evidence for the role of VD treatment in improving various clinical parameters in vitamin D-deficient women with PCOS including glucose intolerance, hypertension, and androgen levels $[43,44]$.

The main finding in the current study explored the impact of VD supplementations $(42,000 \mathrm{IU}$ oral vitamin $\mathrm{D}$ per week and $500 \mathrm{mg}$ calcium carbonate per day for 12 weeks) on serum lipids and glucose parameters.

The current study revealed that in the PCOS group, after VD supplementation, there were significant improvements of glycemic parameters including FPG, FSI, HOMA $\beta$, and HOMA-IR, compared to PCOS women at baseline of the study as well as an improvement of obesity indices BMI and WHR.

Similar results observed by Pal et al. found that VD supplementation has been shown to improve blood pressure and decrease IR, total testosterone, and androstenedione levels in vitamin D-deficient women with PCOS [43].

Supporting our results, in a study by Wehr et al., PCOS women receiving weekly 20,000 IU of vitamin D3 for 24 weeks demonstrated a significant decrease in triglycerides at 12 weeks and 24 weeks [33].

Our findings are in concordance with Kotsa et al. showed that triglyceride level significantly decreased and HDL 
significantly increased in 15 obese women with PCOS after they received alfacalcidol $1 \mathrm{~g} / \mathrm{d}$ for 3 months [45].

Regarding the PCOS phenotype, androgenic profile, and inflammatory markers, there were significantly decreased values after the intervention. Even more important, 25(OH)-D serum levels were increased after VD supplementations.

Interestingly, Yildizhan et al. found that the administration of high doses of vitamin $\mathrm{D}$ led to the attenuation of hyperandrogenism and menstrual disturbances in women with PCOS. Moreover, treatment of low concentrations of 25(OH)-D should be kept in mind during managing obese women with PCOS [42].

Similar results were described in Selimoglu et al. study conducted on PCOS patients vitamin D supplementation has been shown to improve cardio-metabolic risks and androgenic profile in vitamin D-deficient women with PCOS [44].

Against our results, Pal and colleagues demonstrated that 3 months of daily oral supplementation of vitamin D (8533

IU) and calcium (530 mg) did not influence IR [43].

Furthermore, regarding the lipid profile, we observed that supplementation with VD had significant effects on the lipid profile.

In our research, we investigated PCOS women for assessment and the influence of VD supplementation on inflammatory biomarkers; in hs-CRP and neutrophil count, there was a highly significant decrease of inflammatory biomarker Moreover, logistic regression analysis was performed to detect predictors associated with VD supplementation among PCOS cases. Of note, logistic regression analysis revealed that among clinical and laboratory features of women with PCOS, androstenedione, FSI, and Hirsutism score were independent predictors of response to VD supplementation.

The only dropout of our study was that not all the enrolled patients in our study complete the trial as the final total number of women enrolled in this study was 55 patients of the intervention group and 40 PCOS patients of the non-intervention group. Thus, we excluded all subjects who did not complete this study. Of note, there were no adverse effects of VD supplementation during the intervention.

\section{The limitations of the study}

As we mention previously in the present study, VD levels vary according to season and region; thus, we should investigate our study in different seasons and should be multicenter to obtain more accurate results, but we will do this in the next studies.

\section{Conclusion}

We found that serum levels of 25(OH)-D were decreased in PCOS patients; moreover, it is negatively correlated to measures of adiposity, insulin resistance, and hs-CRP as well as clinical and laboratory markers of PCOS. Stepwise multiple linear regression analysis revealed that CIMT, FSI, and FPG were the main predictors of 25(OH)-D levels among other clinical and laboratory biomarkers. Considering the controversy about the effects of VD supplementation on inflammatory and metabolic disorders, our novel findings revealed the favorable effect of VD supplementation on an inflammatory biomarker, clinical feature, and androgenic profile of PCOS as well as cardio-metabolic risk factors. Furthermore, VD supplementation could be a promising treatment of PCOS and its associated morbidity in PCOS-deficient women.

\section{Abbreviations}

25(OH)-D: 25 Hydroxy cholecalciferol; CIMT: Carotid intima-media thickness; FPG: Fasting plasma glucose; FSI: Fasting serum insulin; HOMA-IR: The homeostatic model assessment-IR; hs-CRP: Serum high-sensitivity C-reactive protein; PCOS: Polycystic ovary syndrome; SHBG: Sex hormone-binding globulin; VD: Vitamin D

\section{Acknowledgements}

Not applicable

Authors' contributions

NMR, AHA, MEL, and HGA collected patients' samples and clinical data. RMA prepared the sample for laboratory investigations. NMR wrote the paper. Statistical analysis, interpretation of data, and preparation of the paper for submitting international were done by NMR. Critical revision of the manuscript was performed by all of the authors. All authors participated in the design of the manuscript, drafted the manuscript, revised the manuscript critically, and approved the final version.

\section{Funding}

No fund

Availability of data and materials

Data available on demand

Ethics approval and consent to participate

Written informed consent was taken from all of the participants after explaining details and benefits as well as risks to them. The ethical committee of the Faculty of Medicine, Zagazig University, approved this study.

Consent for publication

Not applicable

\section{Competing interests}

The authors declare that they have no competing interests.

\section{Author details}

${ }^{1}$ Internal Medicine Department, Faculty of Medicine, Zagazig University, Zagazig, Egypt. ${ }^{2}$ Obstetrics and Gynecology Department, Faculty of Medicine, Zagazig University, Zagazig, Egypt. ${ }^{3}$ Cardiology Department, Faculty of Medicine, Zagazig University, Zagazig, Egypt. ${ }^{4}$ Clinical Pathology Department, Faculty of Medicine, Zagazig University, Zagazig, Egypt.

Received: 2 July 2019 Accepted: 9 October 2019

Published online: 12 November 2019

References

1. Franks S, Robinson S, Willis DS (1995) Polycystic ovary syndrome. N Engl J Med 333:883-861

2. Dunaif A (1997) Insulin resistance and the PCOS: mechanisms and implication for pathogenesis. Endocr Rev 18:774-800 
3. Lorenz LB, Wild RA (2007) Polycystic ovarian syndrome: an evidence-based approach to evaluation and management of diabetes and cardiovascular risks for today's clinician. Clin Obstet Gynecol 50:226-243

4. Talbott E, Guzick D, Clerici A, Berga S, Detre K, Weimer K et al (1995) Coronary heart disease risk factors in women with polycystic ovary syndrome. Arterioscler Thromb Vasc Biol 15:821-826

5. Victor VM, Rocha M, Bañuls C, Alvarez A, de Pablo C, Sanchez-Serrano M et al (2011) Induction of oxidative stress and human leukocyte/endothelial cell interactions in polycystic ovary syndrome patients with insulin resistance. J Clin Endocrinol Metab 96(10):3115-3122

6. Bikle D (2009) Nonclassic actions of vitamin D. J Clin Endocrinol Metab 94(1):26-34

7. Holick MF (2002) Vitamin D. the underappreciated D-lightful hormone that is important for skeletal and cellular health. Curr Opin Endocrinol Diabetes 9:87-98

8. Hahn S, Haselhorst U, Tan S, Quadbeck B, Schmidt M, Roesler S et al (2006) Low serum 25-hy-droxyvitamin D concentrations are associated with insulin resistance and obesity in women with polycystic ovary syndrome. Exp Clin Endocrinol Diabetes 114(10):577-583

9. Schmidt-Trucksass A, Grathwohl D, Schmid A, Boragk R, Upmeier C, Keul J, Huonker M (1999) Structural, functional, and hemodynamic changes of the common carotid artery with age in male subjects. Arterioscler Thromb Vasc Biol 19:1091-1097

10. Bianchini E, Bozec E, Gemignani V, Faita F, Giannarelli C, Ghiadoni L, Demi M, Boutouyrie P, Laurent S (2010) Assessment of carotid stiffness and intima-media thickness from ultrasound data: comparison between two methods. J Ultrasound Med 29:1169-1175

11. The Rotterdam ESHRE-ASRM-Sponsored PCOS Consensus Workshop Group (2004) Revised 2003 consensus on diagnostic criteria and long-term health risks related to polycystic ovary syndrome (PCOS). Hum Reprod 19:41-47

12. Ferriman D, Gallwey JD (1961) Clinical assessment of body hair growth in women. J Clin Endocrinol Metab 21:1440-1447

13. Friedewald WT, Levy RI, Fredrickson DS (1972) Estimation of the concentration of low-density lipoprotein cholesterol in plasma, without use of the preparative ultracentrifuge. Clin Chem 18:499-502

14. Matthews DR, Hosker JP, Rudenski AS et al (1985) Homeostasis model assessment: insulin resistance and beta-cell function from fasting plasma glucose and insulin concentrations in man. Diabetologia 28:412-419

15. Holick MF (2007) Vitamin D deficiency. N Engl J Med 357:266-281

16. Stein JH, Korcarz CE, Hurst RT et al (2008) Use of carotid ultrasound to identify subclinical vascular disease and evaluate cardiovascular disease risk: a consensus statement from the American Society of Echocardiography Carotid Intima-Media Thickness Task Force. Endorsed by the Society for Vascular Medicine. J Am Soc Echocardiogr 21:93-111. https://doi.org/10.1016/.echo.2007.11.011

17. Gordon D, Haynes C, Blumenfeld J, Finch SJ (2005) PAWE-3D: visualizing power for association with error in case/control genetic studies of complex traits. Bioinformatics 21:3935-3937

18. Goodarzi MO, Dumesic DA, Chazenbalk G, Azziz R (2011) Polycystic ovary syndrome. Etiology, pathogenesis and diagnosis. Nat Rev Endocrinol 7:219-223

19. Brassard M, AinMelk Y, Baillargeon JP (2008) Basic infertility including polycystic ovary syndrome. Med Clin North Am 92:1163-1192

20. Rashad NM, El-Shal AS, Amin Al, Soliman MH (2017) Effects of probiotics supplementation on macrophage migration inhibitory factor and clinical laboratory feature of polycystic ovary syndrome. J Funct Foods 36:317-324

21. Rashad NM, El-Shal AS, Abdelaziz AM (2013) Association between inflammatory biomarker serum procalcitonin and obesity in women with polycystic ovary syndrome. J Reprod Immunol 97(2):232

22. El-Shal AS, Shalaby SM, Aly NM, Rashad NM, Abdelaziz AM (2013) Genetic variation in the vitamin D receptor gene and vitamin D serum levels in Egyptian women with polycystic ovary syndrome. Mol Biol Rep 40(11):6063-6073

23. Glueck CJ, Woo JG, Khoury PR, Morrison JA, Daniels SR, Wang P (2015) Adolescent oligomenorrhea (age 14-19) tracks into the third decade of life (age 20-28) and predicts increased cardiovascular risk factors and metabolic syndrome. Metabolism 64:539-553

24. Zhao L, Zhu Z, Lou H, Zhu G, Huang W, Zhang S, Liu F (2016) Polycystic ovary syndrome (PCOS) and the risk of coronary heart disease (CHD): a meta-analysis. Oncotarget 7:33715-33721. https://doi.org/10.18632/oncotarget.9553

25. Morgan CL, Jenkins-Jones S, Currie CJ, Rees DA (2012) Evaluation of adverse outcome in young women with polycystic ovary syndrome versus matched, reference controls: a retrospective, observational study. J Clin Endocrinol Metab 97:3251-3260
26. Wild RA, Rizzo M, Clifton S, Carmina E (2011) Lipid levels in polycystic ovary syndrome: systematic review and meta-analysis. Fertil Steril 95:1073-1079

27. Al Mulhim AA, Abul Heija AA, Al Talib AA, Al Turki HA, Gasim TG (2013) Hormonal, metabolic and clinical profile of Saudi women with polycystic ovary syndrome. Saudi J Med Med Sci 1(1):30-34. https://doi.org/10.4103/1658-631X.112920

28. Riachy R, Vandewalle B, Kerr Conte J, Moerman E, Sacchetti P, Lukowiak B, Gmyr V, Bouckenooghe T, Dubois M, Pattou F (2002) 1,25-dihydroxyvitamin D3 protects RINm5F and human islet cells against cytokine-induced apoptosis: implication of the antiapoptotic protein A20. Endocrinology 143: 4809-4819

29. Gysemans CA, Cardozo AK, Callewaert H, Giulietti A, Hulshagen L, Bouillon R, Eizirik DL, Mathieu C (2005) 1,25-Dihydroxyvitamin D3 modulates expression of chemokines and cytokines in pancreatic islets: implications for prevention of diabetes in nonobese diabetic mice. Endocrinology 146:1956-1964

30. Van Etten E, Mathieu C (2005) Immunoregulation by 1,25-dihydroxyvitamin D3: basic concepts. J Steroid Biochem Mol Biol 97:93-101

31. D'Ambrosio D, Cippitelli M, Cocciolo MG, Mazzeo D, Di Lucia P, Lang R, Sinigaglia F, Panina-Bordignon P (1998) Inhibition of IL-12 production by 1,25-dihydroxyvitamin D3. Involvement of NF-kappaB downregulation in transcriptional repression of the p40 gene. J Clin Invest 101:252-262

32. Pittas AG, Joseph NA, Greenberg AS (2004) Adipocytokines and insulin resistance. J Clin Endocrinol Metab 89:447-452

33. Wehr E, Pilz S, Schweighofer N et al (2009) Association of hypovitaminosis D with metabolic disturbances in polycystic ovary syndrome. Eur J Endocrinol 161(4):575-582

34. Hahn S, Haselhorst U, Tan S et al (2006) Lowserum25-hydroxyvitamin D concentrations are associated with insulin resistance and obesity in women with polycystic ovary syndrome. Exp Clin Endocrinol Diabetes 114(10):577-583

35. Jia X-Z, Wang Y-M, Zhang N, Guo L-N, Zhen X-L et al (2015) Effect of vitamin $\mathrm{D}$ on clinical and biochemical parameters in polycystic ovary syndrome women: a meta-analysis. Obstet Gynaecol Res 41(11):1791-1802. https://doi.org/10.1111/jog.12793

36. Panidis D, Balaris C, Farmakiotis D, Rousso D, Kourtis A, Balaris V et al (2005) Serum parathyroid hormone concentration are increased in women with polycystic ovary syndrome. Clin Chem 51(9):1691-1697

37. Li HW, Brereton RE, Anderson RA, Wallace AM, Ho CK (2011) Vitamin D deficiency is common and associated with metabolic risk factors in patients with polycystic ovary syndrome. Metab Clin Exp 60:1475-1481.

38. Zhang FF, Al Hooti S, Al Zenki S, Alomirah H, Jamil KM, Rao A, Al Jahmah N, Saltzman E, Ausman LM (2015) Vitamin D deficiency is associated with high prevalence of diabetes in Kuwaiti adults: results from a national survey. BMC Public Health 16:100

39. Bland R, Markovic D, Hills CE, Hughes SV, Chan SL, Squires PE et al (2004) Expression of 25-hydroxyvitamin D3-1alpha-hydroxylase in pancreatic islets. J Steroid BiochemMol Biol 89-90(1-5):121-125

40. Milner RD, Hales CN (1967) The role of calcium and magnesium in insulin secretion from rabbit pancreas studied in vitro. Diabetologia 3(1):47-49. https://doi.org/10.1007/BF01269910

41. Velija-Asimi Z (2014) Evaluation of the association of vitamin D deficiency with gonadotropins and sex hormone in obese and non-obese women with polycystic ovary syndrome. Med Glas 11:170-176

42. Yildizhan R, Kurdoglu M, Adali E, Kolusari A, Yildizhan B, Sahin HG, Kamaci M (2009) Serum 25-hydroxyvitamin D concentrations in obese and non-obese women with polycystic ovary syndrome. Arch Gynecol Obstet 280:559-563

43. Pal L, Berry A, Coraluzzi L, Kustan E, Danton C, Shaw J, Taylor H (2012) Therapeutic implications of vitamin D and calcium in overweight women with polycystic ovary syndrome. Gynecol Endocrinol 28:965-968

44. Selimoglu H, Duran C, Kiyici S et al (2010) The effect of vitamin D replacement therapy on insulin resistance and androgen levels in women with polycystic ovary syndrome. J Endocrinol Investig 33(4):234-238

45. Kotsa K, Yavropoulou MP, Anastasiou O, Yovos JG (2009) Role of vitamin D treatment in glucose metabolism in polycystic ovary syndrome. Fertil Steril 92(3):1053-1058

\section{Publisher's Note}

Springer Nature remains neutral with regard to jurisdictional claims in published maps and institutional affiliations. 